\title{
Models of Normal Variation and Local Contrasts in Hippocampal Anatomy*
}

\author{
Xinyang $\mathrm{Liu}^{1}$, Washington Mio ${ }^{1}$, Yonggang $\mathrm{Shi}^{2}$, Ivo Dinov ${ }^{2}$, Xiuwen $\mathrm{Liu}^{3}$, \\ Natasha Leporé ${ }^{2}$, Franco Leporé ${ }^{4}$, Madeleine Fortin ${ }^{4}$, Patrice Voss ${ }^{4}$, \\ Maryse Lassonde ${ }^{4}$, and Paul M. Thompson ${ }^{2}$ \\ ${ }^{1}$ Department of Mathematics, Florida State University, Tallahassee, FL 32306 \\ ${ }^{2}$ Laboratory of Neuro Imaging, UCLA School of Medicine, Los Angeles, CA 90095 \\ ${ }^{3}$ Department of Computer Science, Florida State University, Tallahassee, FL 32306 \\ ${ }^{4}$ Departement de Psychologie, Université de Montréal, Montréal, QC, Canada
}

\begin{abstract}
We develop a model of continuous spherical shapes and use it to analyze the anatomy of the hippocampus. To account for the geometry of bends and folds, the model relies on a geodesic metric that is sensitive to first-order deformations. We construct an atlas of the hippocampus as a mean shape and develop statistical models to characterize quantitative and qualitative normal shape variation. We also develop a localization tool to identify local contrasts in the anatomy of different populations. The tool is applied to the detection, characterization and visualization of anatomical differences such as local enlargement and gains in volume on the right hippocampus of blind subjects.
\end{abstract}

\section{Introduction}

The construction of anatomical atlases is of primary interest in computational anatomy. An atlas provides a reference geometry that can aid in the development of models of normal anatomical variation and in the detection and characterization of differences between populations and abnormal changes associated with diseases. Structural and functional brain data of an individual are recorded on a geometry that is specific to the individual, but upon registration with an atlas, data for a population can be pooled over a common domain for comparison and analysis. Thus, the geometry of an atlas should reflect the anatomical characteristics shared by the members of a population. This suggests that it be constructed as a "mean" shape and estimated from samples of a representative cross-section. From a modeling standpoint, this poses the problems of defining mean shapes and developing a framework for algorithmic shape representation and analysis. This brings us to shape spaces.

The first formal treatment of shapes, due to Kendall [1, is based on a landmark representation of shapes and on a metric based solely on the coordinates of the landmark points. Since typical meshes employed in brain mapping are

\footnotetext{
* This research was supported in part by NSF grants DMS-0713012 and CCF-0514743, and NIH Roadmap for Medical Research grant U54 RR021813.
}

D. Metaxas et al. (Eds.): MICCAI 2008, Part II, LNCS 5242, pp. 407 415, 2008.

(C) Springer-Verlag Berlin Heidelberg 2008 
rather dense, in neuro-imaging, it is more natural to construct spaces of continuous shapes and use discrete approximations in computations. Moreover, to effectively encode differences in bending and folding patterns, we employ a measure of shape dissimilarity that explicitly accounts for first-order differences with the use of Sobolev-type metrics. In this setting, we propose a fast algorithm for the computation of mean shapes, which is a variant of an algorithm of Huckemann and Ziezold, which was developed for the study of planar shapes in classical landmark theory [2]. This framework allows us to use tangent-space statistics at the mean shape [3] to develop models of shape variation from samples. Another important facet of the analysis of biomedical shapes is the need to identify the regions where the most significant anatomical differences occur, for example, to characterize the statistically significant group differences. Although shape distance is a global measure of shape divergence, it is related to the minimum energy required to deform shapes. As this energy is an integration of local contributions, we can define an energy density function and use it as a localization tool that lends the desired local-global character to the shape model.

As an application, we construct an atlas of the surface of the right hippocampus based on a control group of 28 subjects. The mean-shape algorithm is used in conjunction with the direct mapping techniques of 4 to register the various surfaces and the methods of [5]6] to obtain parametric representations. We construct a "Gaussian" model using tangent-space principal component analysis, which allows us to quantify normal anatomical variation and visualize the main modes of variation. We also generate random samples from the model as supporting evidence of its validity. We employ the localization tool to characterize and visualize the statistically significant differences between the control group and a group of 22 blind subjects, as detected by the model. This type of study is motivated by evidence that blind individuals exhibit above-normal spatial and auditory abilities (cf. [7]). In consonance with this evidence, our model indicates local enlargement in specific areas of the hippocampus. Other morphological studies of the brain with different techniques include [8,1011].

\section{Spaces of Spherical Shapes}

We develop a model of "continuous" spherical shapes in $k$-dimensional Euclidean space $\mathbb{R}^{k}$. The unit sphere $\mathbb{S}^{2}$ in $3 \mathrm{D}$ space centered at the origin will be used as the reference domain. A spherical shape will be represented by a mapping $\alpha: \mathbb{S}^{2} \rightarrow \mathbb{R}^{k}$. If $\alpha$ and $\beta$ are two such mappings, the parameter $p \in \mathbb{S}^{2}$ indexes a correspondence between the shapes, where we think of $(p, \alpha(p))$ and $(p, \beta(p))$ as corresponding points. Thus, built-in to this representation is the assumption that shape correspondences have been established.

To define a shape metric that accounts for first-order differences, we look at the differential of $\alpha$. If $T_{p} \mathbb{S}^{2}$ is the tangent space (plane) to $\mathbb{S}^{2}$ at $p$, the differential of $\alpha$ at $p$ is the linear mapping $d \alpha_{p}: T_{p} \mathbb{S}^{2} \rightarrow \mathbb{R}^{k}$ such that $d \alpha_{p}(v)$ is the directional derivative of $\alpha$ along $v$. If $A, B: T_{p} \mathbb{S}^{2} \rightarrow \mathbb{R}^{k}$ are linear mappings, define the inner product $\langle A, B\rangle=\operatorname{trace}\left(B^{*} A\right)$, where $B^{*}: \mathbb{R}^{k} \rightarrow T_{p} \mathbb{S}^{2}$ is the adjoint of $B$. 
If $\left\{e_{1}, e_{2}\right\}$ is an orthonormal basis of $T_{p} \mathbb{S}^{2}$, the inner product can be calculated as $\langle A, B\rangle=A\left(e_{1}\right) \cdot B\left(e_{1}\right)+A\left(e_{2}\right) \cdot B\left(e_{2}\right)$. The quantity $\|A\|=\sqrt{\langle A, A\rangle}$ is the Frobenius norm of $A$. We say that $\alpha: \mathbb{S}^{2} \rightarrow \mathbb{R}^{k}$ has square-integrable derivative if $d \alpha_{p}$ is defined for almost every $p$ and the function $p \mapsto\left\|d \alpha_{p}\right\|^{2}$ is integrable over the 2-sphere.

Our construction of a shape space begins with the vector space $H_{1}^{k}$ of all mappings $\alpha: \mathbb{S}^{2} \rightarrow \mathbb{R}^{k}$ with square-integrable derivative, which we equip with a Sobolev-type metric. Since the case $k=1$ is technically useful, for $k=1$, we often drop the superscript and write $H_{1}=H_{1}^{1}$. To control the sensitivity of the metric to changes in $d \alpha$ due to small variations or noise, or to emphasize a specific range of frequencies or particular scales, we introduce a bounded linear operator $L: H_{1} \rightarrow H_{1}$, which can be a smoothing operator or a band-pass filter among other alternatives. If $\alpha=\left(\alpha_{1}, \ldots, \alpha_{k}\right)$, we let $L \alpha=\left(L \alpha_{1}, \ldots, L \alpha_{k}\right)$. Given weights $a, b>0$, with $a+b=1$, define the inner product

$$
\langle\alpha, \beta\rangle_{1}^{k}=a \int_{\mathbb{S}^{2}} \alpha(p) \cdot \beta(p) d \sigma(p)+b \int_{\mathbb{S}^{2}}\left\langle d(L \alpha)_{p}, d(L \beta)_{p}\right\rangle d \sigma(p),
$$

where $d \sigma$ denotes the area element of $\mathbb{S}^{2}$. The associated norm is denoted $\|\cdot\|_{1}^{k}$. For $k=1$, we simplify the notation to $\langle,\rangle_{1}$ and $\|\cdot\|_{1}$.

In analogy with the landmark theory of shapes, to obtain a representation that is invariant under translations and scale, we place the centroid of $\alpha$ at 0 and normalize the norm to be unitary. In other words, we require that $\int_{\mathbb{S}^{2}} \alpha(p) d \sigma(p)=0$ and $\|\alpha\|_{1}^{k}=1$. Mappings $\alpha$ satisfying these conditions will be referred to as preshapes. The first condition restricts $\alpha$ to a linear subspace of $H_{1}^{k}$ and the second places $\alpha$ on the unit sphere $S$ of that subspace, which we call the pre-shape space of spherical surfaces. The geodesic distance between the pre-shapes $\alpha, \beta \in S$ is the length of the shortest arc of great circle in $S$ connecting $\alpha$ and $\beta$ and is given by $h(\alpha, \beta)=\arccos \langle\alpha, \beta\rangle_{1}^{k}$.

Next, we look at the action of an orthogonal transformation $U \in O(k)$ on preshapes, which is given by $\alpha \mapsto U \circ \alpha$, where $\circ$ denotes composition of mappings. The orbit $\mathcal{O}_{\alpha}=\{U \circ \alpha: U \in O(k)\}$ consists of all pre-shapes that differ from $\alpha$ by a rigid motion and therefore have the same shape as $\alpha$. Thus, the shape space $\Sigma$ is defined as the quotient space of $S$ under the action of the orthogonal group; that is, $\Sigma=S / O(k)$. If $s_{\alpha}, s_{\beta} \in \Sigma$ are the shapes of $\alpha$ and $\beta$, the shape distance is defined as $d\left(s_{\alpha}, s_{\beta}\right)=\min _{U, V \in O(k)} h(V \circ \alpha, U \circ \beta)$. As orthogonal maps act on $S$ as distance-preserving transformations, one may fix the pre-shape $\alpha$ and calculate the distance as $d\left(s_{\alpha}, s_{\beta}\right)=\min _{U \in O(k)} h(\alpha, U \circ \beta)=\min _{U \in O(k)} \arccos \langle\alpha, U \circ \beta\rangle_{1}^{k}$. The classical Procrustes alignment of configurations of landmarks can be extended to this setting to find the optimal orthogonal transformation $\hat{U}$ that realizes the shape distance. Recall that if the $k \times n$ matrices $P, Q$ represent two centered configurations of $n$ landmarks in $\mathbb{R}^{k}$, the optimal rotational alignment is obtained from a singular value decomposition (SVD) of $P Q^{T}$, whose $(i, j)$-entry is the inner product of the $i$ th row of $P$ and the $j$ th row of $Q$ [1]. This interpretation extends to pre-shapes in $H_{1}^{k}$. If we write the components of a pre-shape as 
$\alpha=\left(\alpha_{1}, \ldots, \alpha_{k}\right)$, a natural analogue of $P Q^{T}$ is the $k \times k$ matrix $A$, whose $(i, j)$ entry is $a_{i j}=\left\langle\alpha_{i}, \beta_{j}\right\rangle_{1}$. One can show that if $A=V_{1} \Sigma V_{2}^{T}$ is an SVD of $A$, then $\hat{U}=V_{1} V_{2}^{T}$ and the shape distance can be calculated as $d\left(s_{\alpha}, s_{\beta}\right)=\omega$, where $\omega=\arccos (\operatorname{tr} \Sigma)$. Letting $\hat{\beta}=\hat{U} \circ \beta$, if $\hat{\beta} \neq \alpha$, then the geodesic deformation is realized by the the path in the pre-shape sphere given by

$$
\Lambda(t)=\cos (\omega t) \alpha+\sin (\omega t) A(\alpha, \beta),
$$

where $0 \leqslant t \leqslant 1$ and $A(\alpha, \beta)=(\hat{\beta}-(\operatorname{tr} \Sigma) \alpha) /\|\hat{\beta}-(\operatorname{tr} \Sigma) \alpha\|_{1}^{k}$. If $\hat{\beta}=\alpha$, then $\Lambda$ is just a constant path. The path may be expressed in terms of the exponential map of the pre-shape sphere at $\alpha$ as $\Lambda(t)=\exp _{\alpha}(\omega t A(\alpha, \beta))$. In particular, $\hat{\beta}=\exp _{\alpha}(\omega A(\alpha, \beta))$.

\section{The Discrete Model}

We just need to describe how the parametric shape representation $\alpha$ and the inner product $\langle\alpha, \beta\rangle_{1}^{k}$ are discretized since the calculation of shape geodesics and shape distances are expressed in terms of these elements. Let $M$ be a triangle mesh in $\mathbb{R}^{3}$ with the topological type of the 2 -sphere. A discrete spherical shape will be represented by a map $\alpha: M \rightarrow \mathbb{R}^{k}$, which is linear on each triangle, so that $\alpha$ is completely determined by its values on the vertex set $V=\left\{v_{1}, \ldots, v_{n}\right\}$ of $M$. Hence, $\alpha$ may be viewed as a $k \times n$ matrix, whose $j$ th column is $\alpha\left(v_{j}\right) \in \mathbb{R}^{k}$. Since the "smoothing" operator $L$ is applied to each component of $\alpha$ independently, in the discrete formulation, it becomes a linear transformation $L: \mathbb{R}^{n} \rightarrow \mathbb{R}^{n}$, which acts on each row of the matrix $\alpha$. We adopt the classical discrete exterior derivative to represent the derivative of $\alpha$, which is defined as follows. Fix an orientation for each edge of $M$ and let $E=\left\{e_{1}, \ldots, e_{m}\right\}$ be the oriented edge set. Then, $d \alpha: E \rightarrow \mathbb{R}^{k}$ is defined by $d \alpha\left(e_{j}\right)=\alpha\left(e_{j}^{+}\right)-\alpha\left(e_{j}^{-}\right)$, where $e_{j}^{+}$and $e_{j}^{-}$ are the terminal and initial vertices of $e_{j}$, respectively. We can fix an orientation for each edge because reversal of orientation of $e_{j}$ simply changes the sign of $d \alpha\left(e_{j}\right)$. Hence, the exterior derivative of $\alpha$ can be viewed as a $k \times m$ matrix, whose $j$ th column is $d \alpha\left(e_{j}\right) \in \mathbb{R}^{k}$.

For each vertex $v_{j}$, let $A_{j}$ be the area of of the star neighborhood of $v_{j}$ in the first barycentric subdivision of $M$. Similarly, for each edge $e_{j}$, we let $B_{j}$ be the sum of the areas of the two triangles formed by $e_{j}$ and the barycenter of the triangles in $M$ having $e_{j}$ as an edge. The discrete inner product is given by

$$
\langle\alpha, \beta\rangle_{1}^{k}=a \sum_{j=1}^{n}\left(\alpha\left(v_{j}\right) \cdot \beta\left(v_{j}\right)\right) A_{j}+b \sum_{j=1}^{m}\left(d(L \alpha)\left(e_{j}\right) \cdot d(L \beta)\left(e_{j}\right)\right) B_{j} .
$$

\section{Mean Shapes and Atlases}

Let $s^{1}, \ldots, s^{r}$ be spherical shapes represented by the pre-shapes $\alpha^{1}, \ldots, \alpha^{r}: \mathbb{S}^{2} \rightarrow$ $\mathbb{R}^{k}$. Given a shape $s$, let $\alpha$ be a pre-shape that represents $s$ and let $U_{i}^{\alpha} \in O(k)$ be 
the orthogonal transformation that optimally aligns $\alpha^{i}$ with $\alpha$. A Fréchet mean shape of the family is a shape that minimizes the total scatter function

$$
V(s)=\frac{1}{2} \sum_{i=1}^{r} d^{2}\left(s, s^{i}\right)=\frac{1}{2} \sum_{i=1}^{r} \arccos ^{2}\left\langle\alpha, U_{i}^{\alpha} \alpha^{i}\right\rangle_{1}^{k} .
$$

This last expression of $V$ shows that we can lift $V$ to a function defined for any $\alpha \in H_{1}^{k}$ since $U_{i}^{\alpha}$ can be defined for any $\alpha$. The estimation of mean shapes can be treated with gradient descent methods, but we take a different approach. We extend to the present context an algorithm of Huckemann and Ziezold for the calculation of mean shapes in the classical landmark theory of planar shapes 2. First, lift $V$ to a function on $H_{1}^{k}$, as indicated above. The unconstrained gradient is given by

$$
\nabla V(\alpha)=-\sum_{i=1}^{r} \frac{\arccos \zeta_{i}(\alpha)}{\sqrt{1-\zeta_{i}^{2}(\alpha)}} U_{i}^{\alpha} \alpha^{i},
$$

where $\zeta_{i}(\alpha)=\left\langle\alpha, U_{i}^{\alpha}\right\rangle$. Since the centering condition is linear, it suffices to use Lagrange multipliers to constrain $\alpha$ to the unit sphere. Thus, at a preshape $\alpha$, we require that $\nabla V(\alpha)=\lambda \alpha$, which makes the gradient perpendicular to the pre-shape sphere. Taking the inner product of (5) with $\alpha$, we get $\lambda=-\sum_{i=1}^{r} \zeta_{i}(\alpha) \arccos \zeta_{i}(\alpha) / \sqrt{1-\zeta_{i}^{2}(\alpha)}$. At a minimum, we obtain $\alpha=$ $\operatorname{sign}(\lambda) \nabla V(\alpha) /\|\nabla V(\alpha)\|_{1}^{k}$. Thus, we are interested in attracting fixed points of the mapping $T: S \rightarrow S$ defined by $T(\alpha)=\operatorname{sign}(\lambda) \nabla V(\alpha) /\|\nabla V(\alpha)\|_{1}^{k}$, as they lead to stable minima of $V$. The strategy adopted in 2 consists of moving towards the fixed point by an iteration of $T$, initializing the procedure with a pre-shape $\alpha$, say, one of the pre-shapes in the given family. For a threshold value $\epsilon>0$, one calculates $T(\alpha), \ldots, T^{n}(\alpha)$ iterating until $\left\|T^{n}(\alpha)-T^{n-1}(\alpha)\right\|_{1}^{k}<\epsilon$. The mean is estimated as the shape associated with the pre-shape $T^{n}(\alpha)$.

We construct an atlas of the right hippocampus with this technique. Triangle meshes representing the right hippocampal surfaces of 28 controls were extracted from MR images. One of the surfaces was fixed and parameterized over $\mathbb{S}^{2}$ with the methods of 56. The remaining 27 were registered with the fixed shape using the direct mapping techniques of [4] and an atlas was constructed as the mean shape of the group. Figure 1 shows 8 of the 28 surfaces in the data set and the atlas constructed with parameter values $a=0.95$ and $b=0.05$. Here, the operator $L$ is the identity. The choice of $a, b$ is discussed below.
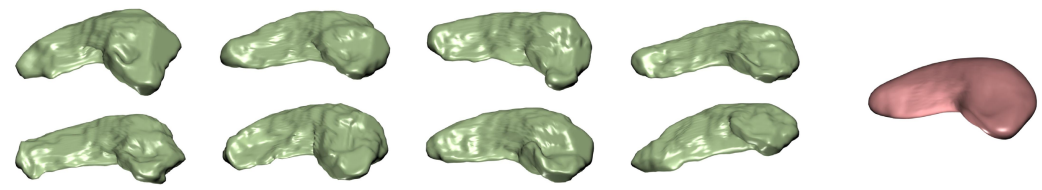

Fig. 1. Eight samples from a control group of 28 subjects and an atlas constructed as the mean shape with $a=0.95$ and $b=0.05$ 


\section{A Model of Normal Shape Variation}

To model normal variation based on the control group of 28 subjects, we employ tangent-space principal component analysis (TPCA) [3] on the pre-shape sphere. Among other things, such construction reveals the main modes of shape variation. Let $\alpha^{i}, 1 \leqslant i \leqslant 28$ be pre-shapes representing the control group and let $\alpha$ represent the mean shape. We assume that each $\alpha^{i}$ has been optimally aligned with $\alpha$. For each $i$, let $V_{i} \in T_{\alpha} S$ be the tangent vector to $S$ at the mean that gets mapped to $\alpha^{i}$ under the exponential map. The vectors $V_{i}$ give a good linear approximation to the data as the samples are fairly concentrated about the mean. Now, we perform PCA on the tangent space $T_{\alpha} S$ with respect to the inner product (3). To recast the results in terms of shapes, we simply exponentiate tangent vectors at $\alpha$ back into pre-shapes. The choice $a=0.95$ and $b=0.05$ for the development of a Gaussian model was based on experimentation with this specific shape data. The decay of the eigenvalues of the covariance matrix and the approximate Gaussianity of the 5-bin histograms of the distributions along the first six principal components, shown in Figure 2, support this choice. To visualize the regions where the most significant shape deformations occur, color plots of the intensity of the deformation fields associated with the first two principal directions are shown on the atlas in Figures 3(a) and 3(b). Figures $4(\mathrm{a})$ and $4(\mathrm{~b})$ show the shape variations along the first two principal directions.

A Gaussian model supported on the subspace corresponding to the six dominant principal components was constructed on the tangent space at the mean. The magnitude of the 7 th eigenvalue falls below $10 \%$ of the largest. In pattern theory, the generation of random samples from a statistical model is proposed as an effective way of testing and validating a model. The idea is that most samples should resemble a normal hippocampus and exhibit variations similar to those observed in the control group. Figure 5 shows six random samples generated from the 6-dimensional Gaussian model.

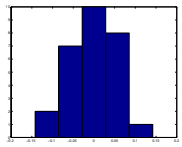

1

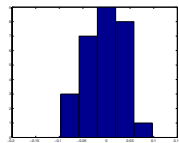

2

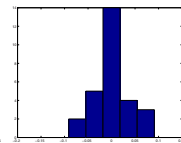

3

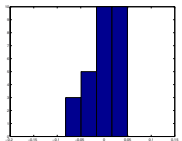

4

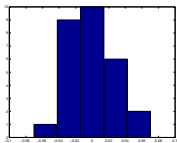

5

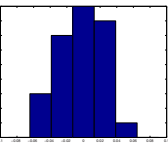

6

Fig. 2. Histograms of the distributions along the first six principal components

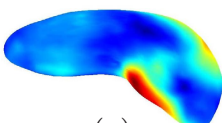

(a)

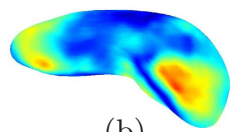

(b)

Fig. 3. Intensity of the deformation fields associated with the first two principal directions shown on the atlas 

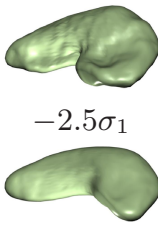

$+0.5 \sigma_{1}$
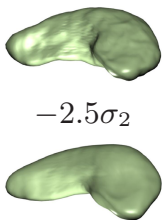

$+0.5 \sigma_{2}$
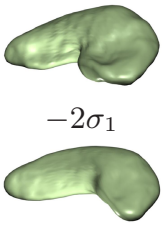

$+\sigma_{1}$

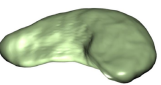

$-2 \sigma_{2}$



$+\sigma_{2}$

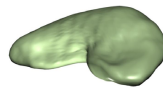

$-1.5 \sigma_{1}$

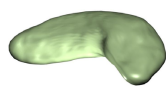

$+1.5 \sigma_{1}$



$-1.5 \sigma_{2}$

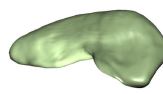

$+1.5 \sigma_{2}$
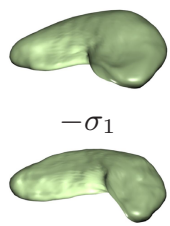

$+2 \sigma_{1}$
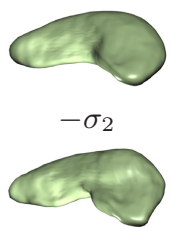

$+2 \sigma_{2}$
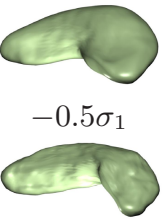

$+2.5 \sigma_{1}$

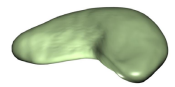

$-0.5 \sigma_{2}$

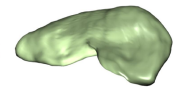

$+2.5 \sigma_{2}$

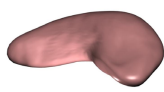

atlas

(a)

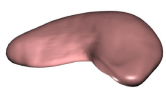

atlas

Fig. 4. Hippocampal shape variation along the first two principal directions
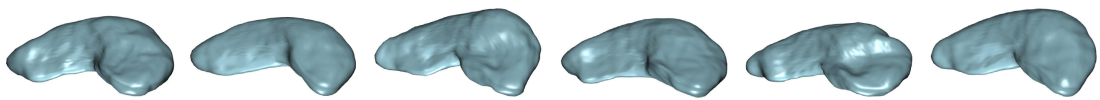

Fig. 5. Random samples from a tangent-space Gaussian model based on the first six principal components

\section{Local Shape Divergence in Early Blindness}

We employ the atlas, the shape metric and the registration techniques of [4] to characterize the statistically significant shape differences observed in early blindness, as modeled on segmented hippocampi of a group of 22 blind subjects. These differences tend to be localized to specific regions, while the shape metric is a global quantifier of dissimilarities. Thus, a direct use of geodesic distances is ineffective. The key observation is that, although the geodesic distance has a global nature, it is directly related to the minimum energy needed to deform a shape into another. This energy is measured assembling pointwise contributions associated with a geodesic deformation field and this allows us to define an energy density function that quantifies the local contributions. A (parametric) geodesic $\Lambda(t), 0 \leqslant t \leqslant 1$, of length $\omega$ is traversed with constant speed $\left\|\partial_{t} \Lambda(t)\right\|_{1}^{k}=\omega$. The energy of the geodesic $\Lambda$, in turn, is given by

$$
\int_{0}^{1}\left(\left\|\partial_{t} \Lambda(t)\right\|_{1}^{k}\right)^{2} d t=\int_{\mathbb{S}^{2}} \int_{0}^{1}\left(a\left\|\partial_{t} \Lambda(p, t)\right\|^{2}+b\left\|d\left(\partial_{t} \Lambda(t)\right)_{p}\right\|^{2}\right) d t d \sigma(p)=\omega^{2}
$$

which shows that the total energy is an integration over $\mathbb{S}^{2}$ of "pointwise energies". Here, to simplify notation, we assumed that the operator $L$ is the identity. This motivates the definition of the energy density function as 


$$
\rho(p)=\frac{1}{\omega^{2}} \int_{0}^{1}\left(a\left\|\partial_{t} \Lambda(p, t)\right\|^{2}+b\left\|d\left(\partial_{t} \Lambda(t)\right)_{p}\right\|^{2}\right) d t
$$

Notice that $\rho$ has been normalized to satisfy $\int_{\mathbb{S}^{2}} \rho(p) d \sigma(p)=1$.

To characterize local shape differences between hippocampal surfaces in the blind and control groups, we aligned all surfaces with the atlas, as indicated in Figure 6(a). We calculated the energy density functions for the geodesics from the atlas to each of the 28 control shapes and the 22 blind subjects. In the discrete representation, $\rho$ becomes a function on the vertex set of the mesh $M$ representing $\mathbb{S}^{2}$. Thus, for each vertex, there is a total of 50 values of energy density. A $t$-test was performed to detect the areas where the statistically significant differences occur. Figure 6(b) highlights the regions where the $p$-values are $<0.05$. Consistent with the above-normal spatial and auditory abilities observed in blind individuals [7, a closer inspection of the geodesic deformation fields reveals that in some of the areas highlighted in Figure 6(b), for most blind subjects, the geodesic fields point outward on the atlas suggesting enlargement and local gains in volume.

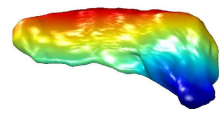

control

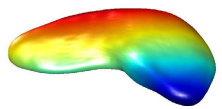

atlas

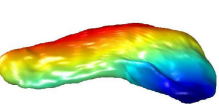

blind

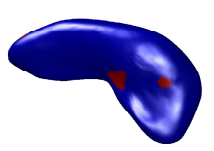

(b)

(a)

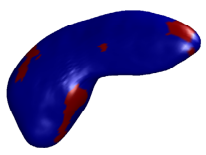

Fig. 6. (a) Examples of registration with the atlas; (b) Two views of the hippocampal atlas indicating the regions where the difference in local anatomy between the control and the blind groups is statistically significant

\section{References}

1. Kendall, D.G.: Shape manifolds, Procrustean metrics and complex projective spaces. Bulletin of London Mathematical Society 16, 81-121 (1984)

2. Huckemann, S., Ziezold, H.: Principal component analysis for Riemannian manifolds, with an application to triangular shape spaces. Advances in Applied Probability 38, 299-319 (2006)

3. Dryden, I.L., Mardia, K.V.: Statistical Shape Analysis. John Wiley \& Son, Chichester (1998)

4. Shi, Y., Thompson, P., Zubicaray, G., Ross, S., Tu, Z., Dinov, I., Toga, A.: Direct mapping of hippocampal surfaces with intrinsic shape context. Neuro Image 36(3), 792-807 (2007)

5. Praun, E., Hoppe, H.: Spherical parametrization and remeshing. In: ACM SIGGRAPH 2003, pp. 340-349 (2003)

6. Liu, X., Bowers, J., Mio, W.: Parametrization, alignment and shape of spherical surfaces. In: 2nd International Conference on Computer Vision Theory and Applications (VISAPP), vol. 1, pp. 199-206 (2007) 
7. Voss, P., Lassonde, M., Gougoux, F., Fortin, M., Guillemot, J.P., Lepore, F.: Earlyand late-onset blind individuals show supra-normal auditory abilities in far-space. Current Biology 14(19), 1734-1738 (2004)

8. Wang, L., Miller, J., Gado, M., Mckeel, D., Rothermich, M., Miller, M., Morris, J., Csernansky, J.: Abnormalities of hippocampal surface structure on very mild dementia of the alzheimer type. Neuro Image 30(1), 52-60 (2006)

9. Gerig, G., Styner, M., Jones, D., Weinberger, D., Lieberman, J.: Shape analysis of brain ventricles using SPHARM. In: Proc. of MMBIA, pp. 171-178 (2001)

10. Styner, M., Gerig, G., Lieberman, J., Jones, D., Weinberger, D.: Statistical shape analysis of neuroanatomical structures based on medial models. Med. Image Anal. 7(3), 207-220 (2003)

11. Pizer, S., Fritsch, D., Yushkevich, P., Johnson, V., Chaney, E.: Segmentation, registration and measurement of shape variation via image object shape. IEEE Trans. Med. Imag. 18(10), 851-865 (1999) 